\title{
Low-molecular-weight polysaccharides from Agaricus blazei Murrill modulate the Th1 response in cancer immunity
}

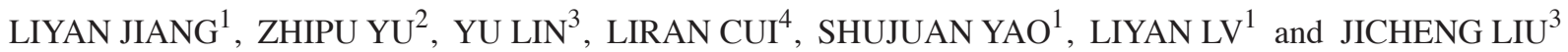 \\ ${ }^{1}$ Department of Pathogen Biology, Qiqihar Medical University; ${ }^{2}$ Department of Medical Research, \\ The Second Affiliated Hospital, Qiqihar Medical University; ${ }^{3}$ Department of Pharmacology, \\ Qiqihar Medical University, Qiqihar, Heilongjiang 161006; ${ }^{4}$ Department of Medical Research, \\ The First Affiliated Hospital, Qiqihar Medical University, Qiqihar, Heilongjiang 161041, P.R. China
}

Received January 15, 2016; Accepted March 2, 2016

DOI: $10.3892 / 01.2018 .7794$

\begin{abstract}
To assess the effect of low-molecular-weight polysaccharides from Agaricus blazei Murrill (ABP-AW1) as an immunoadjuvant therapy for type 1 T-helper (Th1) responses in tumorigenesis, C57BL/6 mice were inoculated subcutaneously with ovalbumin (E.G7-OVA). After 3, 10 and 17 days, the mice were immunized with PBS, OVA alone, or OVA and ABP-AW1, at low $(50 \mu \mathrm{g})$, intermediate $(100 \mu \mathrm{g})$ or high $(200 \mu \mathrm{g})$ doses. Tumor growth was examined and compared among the groups, as were the following parameters: Splenocyte viability/proliferation, peripheral blood $\mathrm{CD}^{+} / \mathrm{CD}^{+} \mathrm{T}$ cell ratio, serum OVA-specific $\mathrm{IgG} 1$ and $\mathrm{IgG} 2 \mathrm{~b}$, secretion of interleukin (IL)-2 and interferon (IFN) $-\gamma$, and IFN- $\gamma$ production on a single cell level from cultured splenocytes. Tumor growth in mice treated with OVA and ABP-AW1 (100 or $200 \mu \mathrm{g}$ ) was significantly slower, compared with in the other groups at the same time-points. OVA with 100 or $200 \mu \mathrm{g}$ ABP-AW1 was associated with a higher number of total splenocytes, a higher ratio of peripheral blood $\mathrm{CD}^{+} / \mathrm{CD}^{+}$T-lymphocytes, higher serum levels of OVA-specific Th1-type antibody IgG2b and greater secretion of the Th1 cytokines IL-1 and IFN- $\gamma$ from splenocytes. ABP-AW1 is a promising immunoadjuvant therapy candidate, due to its ability to boost the Th1 immune response when co-administered with a cancer vaccine intended to inhibit cancer progression.
\end{abstract}

\section{Introduction}

Four general therapeutic approaches have been developed during the long fight with cancer: Surgery, chemotherapy,

Correspondence to: Dr Jicheng Liu, Department of Pharmacology, Qiqihar Medical University, 333 North Bukui Street, Jianhua, Qiqihar, Heilongjiang 161006, P.R. China

E-mail: y13504526515@126.com

Key words: Agaricus blazei Murrill polysaccharide, ovalbumin, Th1, cancer immunity, immunoadjuvant radiotherapy and immunotherapy (1). Unlike the first three, which target the tumor directly, immunotherapy works indirectly through the immune system (2). The initial evidence demonstrating that immunotherapy could be effective in targeting cancer was from a study published by Coley in 1893 (3). Coley injected streptococcal organisms (Coley's toxin) into patients with cancer, and, in a number of cases, tumor regression was observed, presumably due to the host's immune system (3). Since then, attitudes towards cancer immunotherapy have fluctuated, although efforts by oncologists and immunologists to develop treatments have never ceased $(4,5)$. Recently, immunotherapy has received positive results as a cancer treatment, with clinical successes with blocking antibodies at two immune checkpoints, cytotoxic T-lymphocyte-associated protein 4 and programmed cell death protein 1 , and with chimeric antigen receptor-transduced $\mathrm{T}$ cells (6).

Cancer immunotherapy has adopted two basic strategies: Passive transfer of anticancer monoclonal antibodies and immune-active $\mathrm{T}$ cells, without activating endogenous immunity; and the active stimulation of specific antitumor immunity in patients with cancer (7). The initial step in the second strategy is to develop an efficient cancer vaccine that specifically triggers antitumor T cell responses (8). Although many tumor-specific antigens have been identified, when activated alone, the majority lack a strong immunogenic activity and fail to initiate effective antitumor immunity (9). The key limiting factor is the lack of an optimal adjuvant therapy that could enhance, in terms of the magnitude, quality and duration, specific immune responses to antigens, while exerting minimal toxicity or immune effects of its own (7). Although novel adjuvant therapies, including saponin (10), bacterial DNA (11), and cytokines (12), have been reported, aluminum salts (alum) remain the sole adjuvant therapy for the majority of therapeutic vaccines (13). Alum effectively induces the antibody-producing type 2 T-helper (Th2) response, but has no effect on cellular immunity, including the type $1 \mathrm{Th}$ (Th1) response, and is associated with toxicity from aluminum accumulation (14). Therefore, in cancer immunotherapy research there is a great demand for a non-toxic adjuvant therapy that stimulates Th1 responses. 
Successful anticancer immunity requires the activation of $\mathrm{CD} 8^{+}$and $\mathrm{CD} 4^{+} \mathrm{T}$ cells (15). $\mathrm{CD} 8^{+}$cytotoxic $\mathrm{T}$ cells directly target and destroy tumor cells expressing major histocompatibility complex class I molecules. $\mathrm{CD} 4^{+} \mathrm{T}$ cells, specifically the Th1 cells, orchestrate multiple cell types to eradicate tumor cells through the secretion of Th1 cytokines, including interferon (IFN) $-\gamma$ and interleukin (IL)-2 (15). Cell types affected by $\mathrm{CD} 4^{+} \mathrm{T}$ cells include $\mathrm{CD} 8^{+} \mathrm{T}$ cells, macrophages and natural killer cells (16); therefore, an immunoadjuvant therapy that promotes Th1 responses may benefit the development of antigen-specific antitumor immunity.

Polysaccharides are natural, low-toxicity macromolecules with various biological functions, including immune-modulation (17). In the laboratory, the mechanisms underlying polysaccharides isolated from fungi and plants and their functions, in regulating immune responses, were investigated. The previous study demonstrated that the low-molecular-weight polysaccharides of the fungus Agaricus blazei Murrill, ABP-AW1, function as a potent Th1-immunity-stimulating adjuvant therapy in wild-type Institute for Cancer Research mice (18). In the present study, the functional significance and underlying mechanisms of ABP-AW1 in cancer immunity were examined, using ovalbumin (OVA) as the model tumor antigen.

\section{Materials and methods}

Experimental animals and cells. This study was approved by the Institutional Animal Care and Use Committee of Qiqihar Medical University (Heilongjiang, China). A total of 50 female C57BL/6 mice (6-8 weeks old; body weight, $20 \pm 2 \mathrm{~g}$ ) were purchased from Yisi Experimental Animal Technology Co., Ltd. (Jilin, China). The mice were housed in a specific pathogen-free facility at $24 \pm 1^{\circ} \mathrm{C}$ and $50 \pm 10 \%$ relative humidity under a 12/12 h light/dark cycle, with food and water provided ad libitum.

The E.G7-OVA T-lymphoma cell line, stably expressing full-length OVA, was provided by Xuetao Cao (Second Military Medical University, Shanghai, China). The E.G7-OVA cells were cultured in RPMI-1640 medium containing $10 \%$ fetal calf serum (FCS; both from HyClone; GE Healthcare Life Sciences, Logan, UT, USA) in a $37^{\circ} \mathrm{C}$ incubator with $5 \% \mathrm{CO}_{2}$.

Extraction and isolation of ABP-AWI. The ABP-AW1 polysaccharide was prepared and characterized as previously described (19). Briefly, Agaricus blazei were treated under reflux 3 times with $95 \%$ ethanol at $75^{\circ} \mathrm{C}$ for $6 \mathrm{~h}$ to remove lipids. The residue was extracted 3 times with distilled water at $75^{\circ} \mathrm{C}$ for $3 \mathrm{~h}$. The precipitate was washed, dried and extracted twice with $0.5 \mathrm{M} \mathrm{NaOH}$ solution containing $0.3 \%$ (w/w) $\mathrm{NaBH}_{4}$ at room temperature overnight. After the debris was removed through filtering, the suspension was neutralized with $0.1 \mathrm{M}$ hydrochloric acid and filtered again. The supernatant containing water-soluble polysaccharide was dialyzed, concentrated, ethanol-precipitated and dried to generate crude polysaccharide (CABP-AW).

The CABP-AW was re-dissolved in distilled water and centrifuged at $4,000 \mathrm{x} \mathrm{g}$ and $4^{\circ} \mathrm{C}$ for $10 \mathrm{~min}$. The supernatant was applied to a diethylaminoethanol Sepharose Fast
Flow column (Amersham Biosciences; GE Healthcare Life Sciences, Pittsburgh, PA, USA) that had been equilibrated with ultrapure water. After loading the sample, the column was eluted stepwise with $\mathrm{NaCl}$ aqueous solution $(0,0.2,0.4$ and $0.6 \mathrm{M})$ at a flow rate of $4 \mathrm{ml} / \mathrm{min}$. The fractions $(8 \mathrm{ml})$ were collected using a Frac-950 (GE Healthcare Sciences). The polysaccharide was further purified by gel-permeation chromatography on a Sepharose 6 Fast Flow column $(2.6 \times 100 \mathrm{~cm}$; GE Healthcare Life Sciences) eluted with $0.15 \mathrm{M} \mathrm{NaCl}$ at a flow rate of $1 \mathrm{ml} / \mathrm{min}$.

Three polysaccharide fractions, ABP-AW1, ABP-AWA1 and ABP-AWB1, were obtained. The eluted ABP-AW1 was applied to a Sephadex ${ }^{\circledR} \mathrm{G}-25$ column $(2.6 \times 40 \mathrm{~cm}$; GEHealthcare Life Sciences) to remove any salts. Subsequently, ABP-AW1 was collected, dialyzed and lyophilized to obtain purified polysaccharide for use in subsequent experiments.

Analysis by gas chromatography-mass spectrometry revealed that the isolated ABP-AW1 had a backbone consisting of $(1 \rightarrow 6)$-linked- $\beta$-d-galactopyranosyl, $(1 \rightarrow 6)$-linked- $\beta$-d-glucopyranosyl and $(1 \rightarrow 3,6)$-linked$\beta$-d-glucopyranosyl. The backbone terminated with $(1 \rightarrow$ )-linked fucose, arabinose and mannose residues at the $0-3$ position of $(1 \rightarrow 3,6)$-linked- $\beta$-d-glucopyranosyl. These six types of residues were present in the ratio of 29:10:10:6:2:2 (19)

Establishment of xenograft tumors and antitumor immunization. To establish xenograft tumors, E.G7-OVA cells in the log phase of growth were washed once with serum-free RPMI-1640 medium and resuspended in $10 \mathrm{ml}$ fresh serum-free RPMI- 1640 medium at $5 \times 10^{6}$ cells $/ \mathrm{ml}$. A total of $5 \times 10^{5}$ cells were then injected subcutaneously into the right lateral thighs of the mice.

After inoculation with the E.G7-OVA cells, all mice were randomly divided into 5 groups of 10 mice each. On days 3 , 10 and 17 post-inoculation, the mice received a subcutaneous injection of $100 \mu \mathrm{l}$ PBS (for PBS group), $100 \mu \mathrm{l}$ PBS containing $100 \mu \mathrm{g}$ OVA (Sigma-Aldrich; Merck KGaA, Darmstadt, Germany; for OVA-alone group), or $100 \mu \mathrm{g}$ OVA together with 50, 100 or $200 \mu \mathrm{g}$ ABP-AW1 (for low-, intermediate-, or high-dose OVA and ABP-AW1 groups, respectively).

From day 7 after tumor cell inoculation, tumor growth in all groups was monitored every two days by measuring the length (L) and width $(\mathrm{W})$ of the growing nodule, and the volume $(\mathrm{V})$ was calculated as $\mathrm{V}=\left(\mathrm{L} \mathrm{x} \mathrm{W}^{2}\right) / 2$.

On day 28 after tumor inoculation, all mice were euthanized and no mice presented signs of distress, cachexia or ulceration on the tumors. The tumor was isolated and weighed, and other tissues including the spleen and the peripheral blood were collected for further experiments.

MTT assay of splenocyte viability/proliferation. The whole mouse spleen was isolated and dissociated into single cells by grinding against a $70-\mu \mathrm{m}$ nylon mesh. The dissociated cells were resuspended in $3 \mathrm{ml}$ serum-free RPMI-1640 medium and centrifuged at $800 \mathrm{x} \mathrm{g}$ and $4^{\circ} \mathrm{C}$ for $30 \mathrm{~min}$ in order to remove erythrocytes and cellular debris. The splenocytes were washed in serum-free RPMI-1640 medium. They were then resuspended in RPMI-1640 with 10\% FCS, and seeded into 96-well plates (Corning Inc., Corning, NY, USA) in 
triplicate, at $5 \times 10^{5}$ cells $/ 100 \mu \mathrm{l} /$ well, and incubated at $37^{\circ} \mathrm{C}$ in a humidified $5 \% \mathrm{CO}_{2}$ incubator, together with concanavalin A (ConA; $5 \mu \mathrm{g} / \mathrm{ml}$; Sigma-Aldrich; Merck KGaA) or OVA $(10 \mu \mathrm{g} / \mathrm{ml})$.

After $60 \mathrm{~h}, 20 \mu \mathrm{l}$ MTT agent $(5 \mathrm{mg} / \mathrm{ml})$ was added into each well and incubated at $37^{\circ} \mathrm{C}$ for a further $4 \mathrm{~h}$. After centrifugation at $250 \mathrm{x} \mathrm{g}$ and room temperature for $5 \mathrm{~min}$, the supernatant was discarded from each well. Dimethylsulfoxide (150 $\mu \mathrm{l} /$ well) was added into each well to dissolve the formazan crystals. The absorbance was measured using a microplate reader at $570 \mathrm{~nm}$ with a $630 \mathrm{~nm}$ reference. The stimulation index (SI) was calculated based on the following formula: $\mathrm{SI}=$ absorbance value for treated cultures/absorbance value for non-treated cultures.

Measurement of peripheral blood $C D 4^{+}$and $C D 8 \alpha^{+} T$ cells. The peripheral blood $\mathrm{CD}^{+}$and $\mathrm{CD}^{+} \mathrm{T}$ cells were measured viaflow cytometry, as previously described (20). Briefly, peripheral blood from the retro-orbital sinus was collected into anti-coagulant tubes. After the lysis of erythrocytes with red blood cell lysis buffer (BD Pharmingen; BD Biosciences, San Jose, CA, USA), the remaining peripheral blood cells were stained with $20 \mu$ l anti-mouse CD4-fluorescein isothiocyanate antibody (undiluted; cat. no. 553032; BD Pharmingen; BD Biosciences) and $20 \mu 1$ CD8 $\alpha$-phycoerythrin antibody (undiluted; cat. no. 557307; BD Pharmingen; BD Biosciences) diluted in fluorescence-activated cell sorting (FACS) buffer $(0.5 \%$ bovine serum albumin in $\mathrm{PBS}$ ) for $30 \mathrm{~min}$ at room temperature. The cells were then washed with FACS buffer, resuspended in $400 \mu \mathrm{l}$ PBS and analyzed usinga BD FACSCalibur ${ }^{\mathrm{TM}}$ flow cytometer (BD Biosciences).

Measurement of OVA-specific IgG1 and IgG2b in serum. The serum levels of OVA-specific IgG1 and IgG2b were measured using an in-house indirect ELISA as previously described (21). In brief, peripheral blood was collected from the retro-orbital sinus and stored at room temperature for $2 \mathrm{~h}$. Following blood coagulation, the sample was centrifuged at $800 \mathrm{x} \mathrm{g}$ and $4^{\circ} \mathrm{C}$ for $15 \mathrm{~min}$, and the serum supernatant was transferred into a clean Eppendorf tube and stored at $-80^{\circ} \mathrm{C}$ for future use.

For ELISA analysis, 96-well microtiter plates were coated with $100 \mu \mathrm{l}$ OVA solution $(50 \mu \mathrm{g} / \mathrm{ml}$ in $50 \mathrm{mM}$ carbonate-bicarbonate buffer; $\mathrm{pH} 9.6)$ overnight at $4^{\circ} \mathrm{C}$. After 3 washes in PBS with $0.05 \%$ Tween (PBST), the plates were blocked with $200 \mu \mathrm{l}$ of $5 \% \mathrm{FCS} / \mathrm{PBS}$ at $37^{\circ} \mathrm{C}$ for $2 \mathrm{~h}$. After 3 washes with PBST, $100 \mu$ l each diluted serum sample (1:500 dilution) was added into the wells, in triplicate, and incubated for $1 \mathrm{~h}$ at $37^{\circ} \mathrm{C}$. Following another 3 washes with PBST, IgG1 and IgG2b (contained in the serum sample) were detected using horseradish peroxidase-conjugated goat anti-mouse IgG1 (1:5,000 dilution, cat. no. 1070-05) or IgG2b (1:5,000 dilution, cat no. 1090-05) (both from Southern Biotech, Birmingham, AL, USA) at $37^{\circ} \mathrm{C}$ for $1 \mathrm{~h}$. The unbound antibodies were removed by washing 6 times with PBST.

The 3,3',5,5'-tetramethylbenzidine substrate (cat. no. T2885; Sigma-Aldrich; Merck KGaA) was added and incubated for $10 \mathrm{~min}$ at $37^{\circ} \mathrm{C}$ in the dark, following which the reaction was stopped by adding $2 \mathrm{M} \mathrm{H}_{2} \mathrm{SO}_{4}(50 \mu \mathrm{l} /$ well). The optical density was measured at $450 \mathrm{~nm}$ using a microplate reader.

Evaluation of the cytokines present within cultured splenocyte supernatants. The splenocytes were seeded into 96-well tissue culture plates at $1 \times 10^{6}$ cells $/ 100 \mu \mathrm{l} /$ well in triplicate. Cells were treated with OVA (final concentration, $10 \mu \mathrm{g} / \mathrm{ml}$ ) at $37^{\circ} \mathrm{C}$ in a humidified $5 \% \mathrm{CO}_{2}$ incubator. After $48 \mathrm{~h}$, the plates were centrifuged at $250 \times \mathrm{g}$ and $4^{\circ} \mathrm{C}$ for $10 \mathrm{~min}$ and the supernatant was collected for the measurement of secreted IFN- $\gamma$ or IL-2 using mouse ELISA kits (DKW12-2000-096 for IFN-g ELISA kit and DKW12-2020-096 for IL-2 ELISA kit; Dakewe Biotech Co., Ltd., Shenzhen, Guangdong, China), in accordance with the manufacturer's instructions. To measure the production of IFN- $\gamma$ from cultured splenocytes on the single-cell level, a mouse IFN- $\gamma$ ELISPOT kit (DKW22-2000-096s; Dakewe Biotech Co., Ltd.) was used as instructed by the manufacturer.

Statistical analysis. Quantitative data are expressed as the mean \pm standard deviation. Multiple comparisons were performed using ANOVA with Student-Newman-Keuls test as a post hoc test in order to analyze the differences among the groups using SPSS version 17.0 software (SPSS, Inc., Chicago, IL, USA). $\mathrm{P}<0.05$ was considered to indicate a statistically significant difference.

\section{Results}

OVA-induced tumor growth. To examine the in vivo efficacy of ABP-AW1 as an adjuvant therapy to boost the tumor response to a cancer vaccine, OVA was used as the tumor antigen and a subcutaneous xenograft mouse model was established, using OVA-expressing E.G7 T-lymphoma cells.

By monitoring tumor growth after immunization with PBS (control), OVA alone or OVA with various doses of ABP-AW1, it was determined that the effects of OVA by itself, or together with low-dose ABP-AW1 $(50 \mu \mathrm{g})$, were similar to those of the PBS control group ( $\mathrm{P}>0.05$; Fig. 1A). However, OVA combined with intermediate-(100 $\mu \mathrm{g})$ or high-dose $(200 \mu \mathrm{g})$ ABP-AW1 was associated with significantly smaller tumor volumes, relative to the control, starting from day 16 after tumor cell inoculation $(\mathrm{P}<0.05$; Fig. 1A). Consistently, by day 28 , the tumor weights of the OVA with intermediate- or high-dose ABP-AW1 (100 or $200 \mu \mathrm{g}$ ) groups were significantly lower than those of the other three groups [PBS, OVA alone, or OVA with low-dose ABP-AW1 (50 $\mu \mathrm{g})$ group; $\mathrm{P}<0.05]$. The tumor weights of the PBS, OVA-alone, and OVA with low-dose ABP-AW1 (50 $\mu \mathrm{g})$ groups were comparable with each other by day 28 following the inoculation of lymphoma cells ( $\mathrm{P}>0.05$; Fig. 1B).

Splenocyte viability/proliferation. To investigate the effect of ABP-AW1 on the overall immune activity, the viability/proliferation of splenocytes isolated from the different groups of mice in response to a non-specific lymphocyte mitogen, ConA, or the specific tumor antigen, OVA was examined (Fig. 2). ConA and OVA treatments resulted in a greater number of splenocytes in mice of the OVA and ABP-AW1 (100 and $200 \mu \mathrm{g}$ ) groups, compared with the mice of the PBS, 

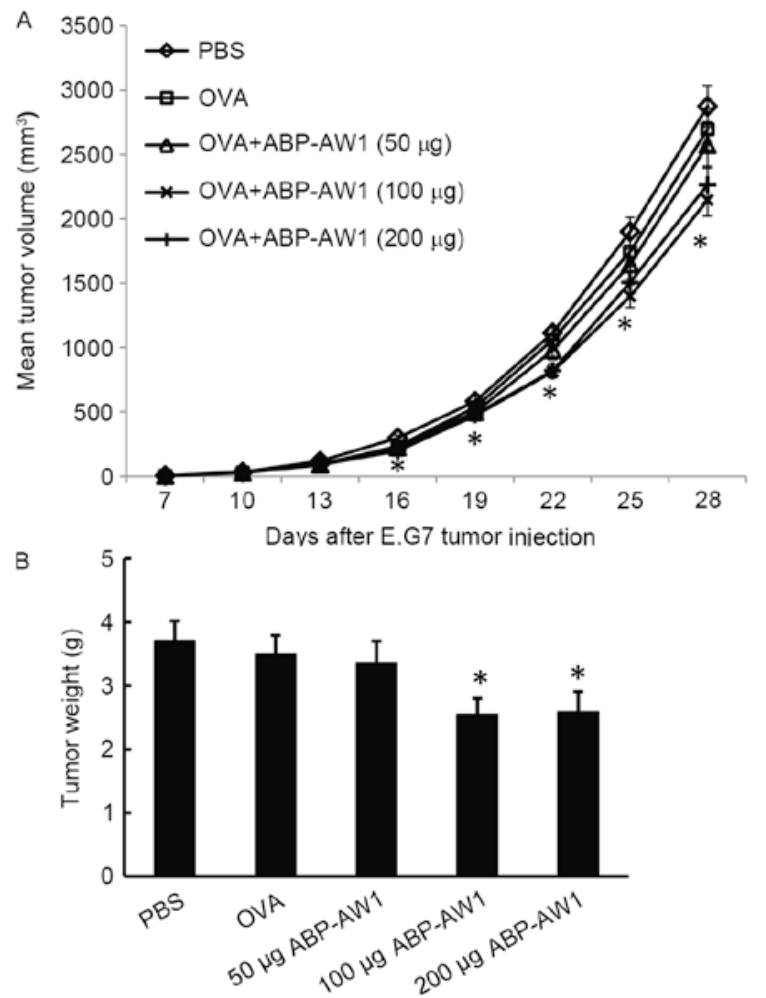

Figure 1. Intermediate- and high-dose ABP-AW1 potentiates the OVA-induced tumor regression. Xenograft tumors were established via the subcutaneous injection of E.G7-OVA cells. Following immunization with PBS, OVA-alone, or OVA plus different doses of ABP-AW1 ( $\mathrm{n}=10 /$ group), the tumor growth was monitored by (A) measuring the length and width of the growing nodule and calculating the tumor volume; or (B) by weighing the tumor on day 28 after tumor cell inoculation. ${ }^{*} \mathrm{P}<0.05$, comparing the OVA and ABP-AW1 (100 or $200 \mu \mathrm{g}$ ) groups to any of the other three groups. OVA, ovalbumin.

OVA-alone, or OVA and ABP-AW1 $(50 \mu \mathrm{g})$ groups $(\mathrm{P}<0.05)$; however, no significant differences were detected among the latter three groups $(\mathrm{P}>0.05)$.

$C D 4^{+} / C D 8^{+} T$-cell ratio in peripheral blood. The ratio of $\mathrm{CD}^{+}$and $\mathrm{CD}^{+} \mathrm{T}$ cells is closely associated with host immune activity. Through flow cytometry analysis, it was determined that the peripheral blood $\mathrm{CD}^{+} / \mathrm{CD}^{+}$ratio of the OVA-alone and OVA and ABP-AW1 (50 $\mu \mathrm{g})$ groups was not significantly different from that of the PBS group $(\mathrm{P}>0.05$, Fig. 3); however, the $\mathrm{CD} 4^{+} / \mathrm{CD}^{+}$ratios of the OVA and ABP-AW1 (100 and $200 \mu \mathrm{g})$ mice were significantly higher than those of the other three groups $(\mathrm{P}<0.05)$. This suggests that intermediate- or high-dose ABP-AW1 notably enhanced the anticancer immune activity of OVA.

Serum levels of OVA-specific IgG2b and IgG1 antibody. Among the serum antibody IgG subtypes, the IgG1 is a marker dominated by Th2-type immune responses, whereas IgG2b is associated with Th1-type immunity $(22,23)$. To analyze which $\mathrm{T}$ cell response is preferentially boosted by ABP-AW1, the serum levels of OVA-specific IgG1 and IgG2b were measured using an ELISA (Fig. 4A). Between all five groups, OVA-specific IgG1 level was not significantly different ( $\mathrm{P}>0.05$; Fig. 4A). The serum IgG2b level, however,
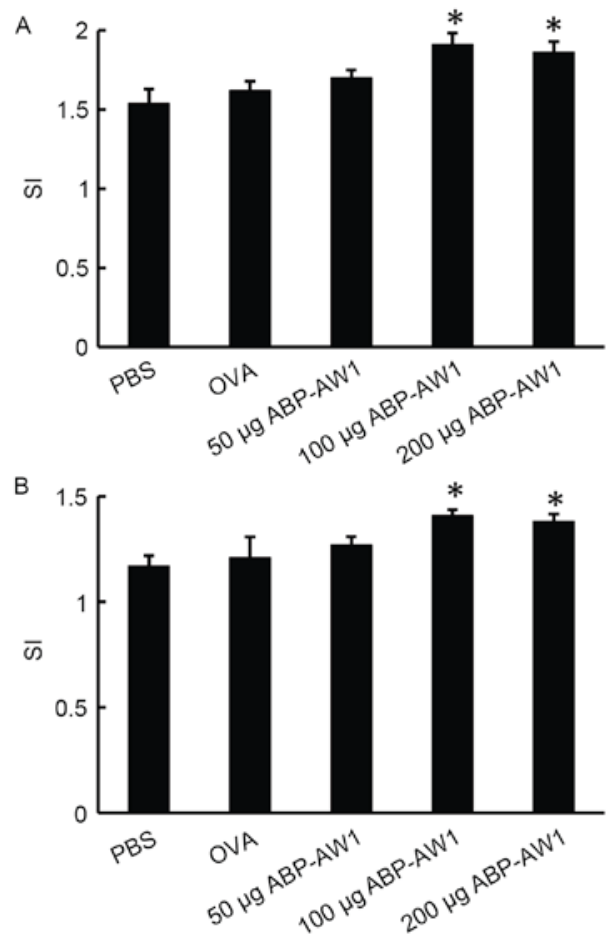

Figure 2. Intermediate and high dose ABP-AW1 promotes splenocyte viability/proliferation in response to ConA or OVA. Splenocytes were isolated from the indicated groups of mice and exposed to (A) ConA or (B) OVA treatment. The splenocyte proliferation was measured by an MTT assay. ${ }^{*} \mathrm{P}<0.05$, as compared with PBS, OVA or OVA and ABP-AW1 (50 $\left.\mu \mathrm{g}\right)$ group. OVA, ovalbumin; ConA, concanavalin A; SI, stimulation index.

was lower than the IgG1 level in the PBS, OVA alone or OVA with low-dose ABP-AW1 $(50 \mu \mathrm{g})$ group, resulting in an IgG2b/IgG1 ratio of $<1$ (Fig. 4B). In contrast, the OVA-specific IgG2b level in the OVA with intermediate or high-dose ABP-AW1 (100 or $200 \mu \mathrm{g}$ ) group was significantly increased, and thus there was a notably higher $\operatorname{IgG} 2 \mathrm{~b} / \operatorname{IgG} 1$ ratio $(>1)$ compared with the other three groups [the PBS, OVA alone, or OVA with low-dose ABP-AW1 $(50 \mu \mathrm{g})$ group; $\mathrm{P}<0.05]$. This indicated that intermediate- and high-dose ABP-AW1 is sufficient to preferentially induce a Th1 response over a Th2 response.

Cytokine production by splenocytes. To further characterize the Th1 responses augmented by ABP-AW1, ELISAs were performed to evaluate the cytokines secreted by cultured splenocytes in response to the specific antigen OVA (Fig. 5). In vivo immunization with OVA and ABP-AW1 (100 or $200 \mu \mathrm{g}$ ) was associated with significantly higher levels of the Th1 cytokines IL-2 (Fig. 5A) and IFN- $\gamma$ compared with those of the other three groups (Fig. 5B; $\mathrm{P}<0.05$ ). The levels of these cytokines in the OVA and ABP-AW1 $(50 \mu \mathrm{g})$ groups were not significantly higher than those in the PBS or OVA-alone group $(\mathrm{P}>0.05)$. The IFN- $\gamma$-producing splenocytes were quantified by ELISPOT analysis, which consistently indicated a significantly higher number of IFN- $\gamma$-positive splenocytes in the OVA and ABP-AW1 (100 and $200 \mu \mathrm{g})$ groups, compared with in the other three groups $(\mathrm{P}<0.05$; Fig. 6); therefore, the data indicate that intermediate-andhigh-dose ABP-AW1 enhances Th1 polarization in the context of cancer development. 

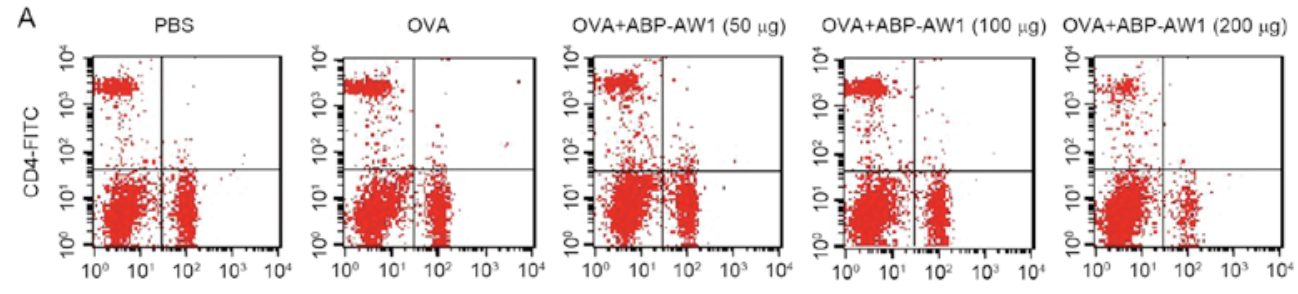

B

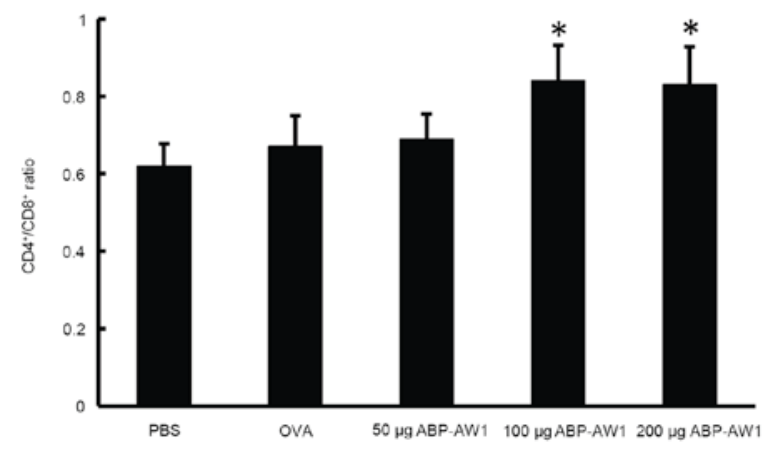

Figure 3. Intermediate and high dose ABP-AW1 increases the $\mathrm{CD}^{+} / \mathrm{CD} 8^{+} \mathrm{T}$ cell ratio in peripheral blood. Peripheral blood was collected from the indicated groups of mice, and $\mathrm{CD}^{+}$and $\mathrm{CD} 8^{+}$lymphocytes were detected by flow cytometry. (A) The representative flow cytometry dot plot from each group, and (B) the quantification of the $\mathrm{CD} 4^{+} / \mathrm{CD} 8^{+}$ratio. ${ }^{*} \mathrm{P}<0.05$, as compared with PBS, OVA or OVA and ABP-AW1 (50 $\mu \mathrm{g}$ ) group. OVA, ovalbumin; FITC, fluorescein isothiocyanate; $\mathrm{CD}$, cluster of differentiation; $\mathrm{PE}$, phycoerythrin.
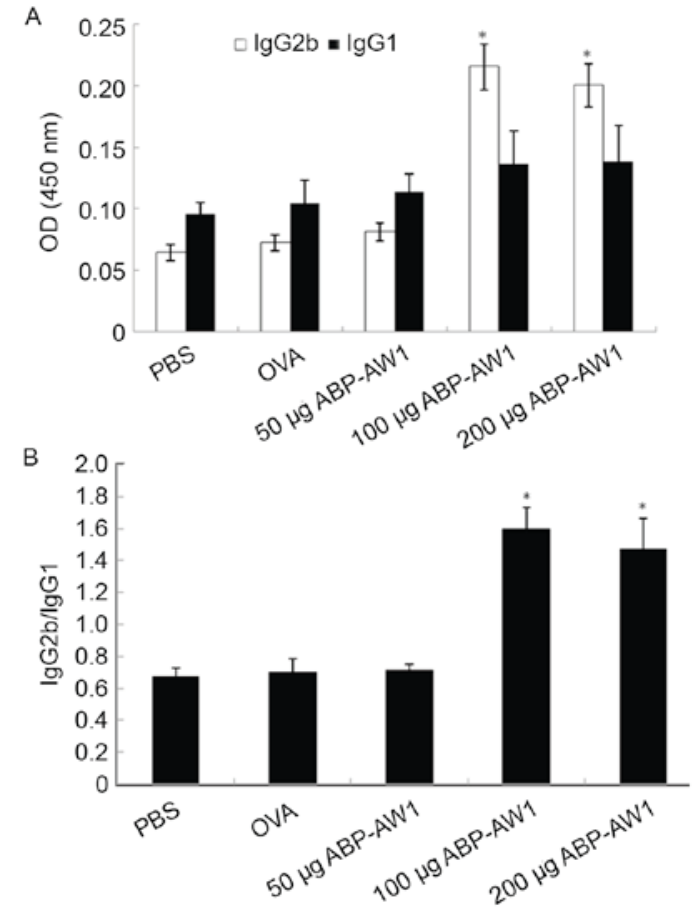

Figure 4. Intermediate and high dose of ABP-AW1 elevates the serum level of OVA-specific IgG2b antibody. The serum level of OVA-specific IgG1 and IgG2b was measured by ELISA from the indicated groups of mice. (A) The OD reading of each antibody and (B) the $\mathrm{IgG} 2 \mathrm{~b} / \mathrm{IgG} 1$ ratio. ${ }^{*} \mathrm{P}<0.05$, as compared with PBS, OVA or OVA and ABP-AW1 (50 $\mu \mathrm{g})$ group. OVA, ovalbumin; OD, optical density.

\section{Discussion}

The present study evaluated the in vivo efficacy of ABP-AW1, isolated from Agaricus blazei, as an immunoadjuvant therapy to boost the anticancer activity of a cancer vaccine, using OVA as the antigen in a mouse model of T-cell lymphoma. The model was created via inoculation with E.G7-OVA cells, and the mice were subsequently treated with PBS, OVA-alone, or OVA together with ABP-AW1 (OVA and ABP-AW1) at low $(50 \mu \mathrm{g})$, intermediate $(100 \mu \mathrm{g})$ or high doses $(200 \mu \mathrm{g})$. By all measures (tumor growth, splenocyte viability/proliferation, peripheral blood $\mathrm{CD} 4^{+} / \mathrm{CD} 8^{+} \mathrm{T}$-lymphocytes, serum OVA-specific IgG2b, splenocyte secretions of IL-2 and IFN- $\gamma$ ), it was observed that ABP-AW1 at intermediate and high doses was an efficacious immunoadjuvant therapy, relative to the other treatment groups.

The development of cancer has generally been associated with six biological traits: Self-sufficiency in growth signals, insensitivity to anti-growth signals, evading apoptosis, limitless replicative potential, sustained angiogenesis, and tissue invasion and metastasis (24). In the last decade, two hallmarks were added to the list: Reprogramming energy metabolism; and evading immune destruction (25). The ability of a tumor to suppress the immune system responses and evade immune destruction supports the importance of cancer immunotherapy. The identification of tumor-associated antigens and of epitopes specifically recognized by distinct $\mathrm{T}$ cell subtypes has greatly stimulated the development of cancer vaccines. However, tumor-associated antigens or epitopes are predominantly antigenic and not immunogenic, with minimal induction of anticancer immunity (26). This justifies the incorporation of an immunoadjuvant therapy as a key component in cancer vaccines (27).

The host's immune system utilizes multiple mechanisms to attempt to control or eradicate cancer, mainly through T-lymphocytes, natural killer cells and macrophages. By contrast, humoral immunity involving antibody production is not a key factor in anticancer immunity, and may 

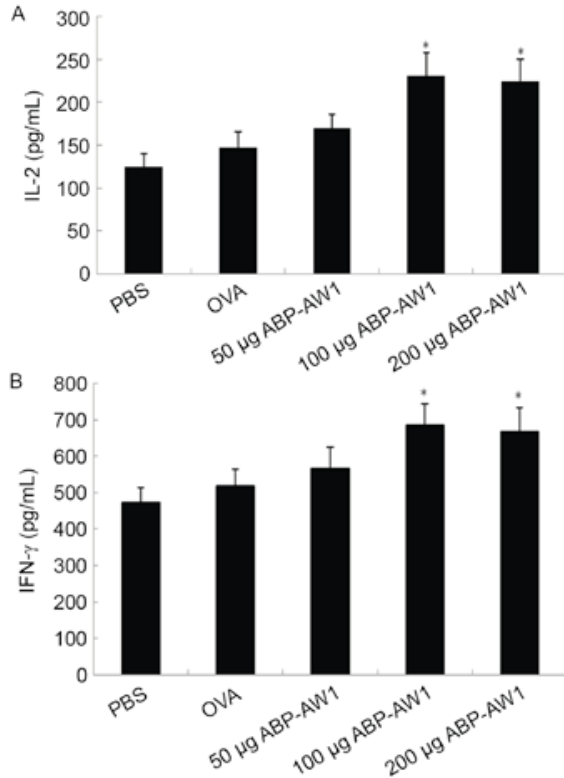

Figure 5. Intermediate and high dose of ABP-AW1 stimulates cytokine secretion by splenocytes in response to OVA. Splenocytes isolated from indicated groups of mice were stimulated with OVA. The secretion of (A) IL-2 and (B) IFN- $\gamma$ into the culture medium was determined by an ELISA. ${ }^{*} \mathrm{P}<0.05$, as compared with PBS, OVA or OVA and ABP-AW1 (50 $\mu \mathrm{g})$ group. OVA, ovalbumin; IL, interleukin; IFN, interferon.

paradoxically promote tumor growth. Among the distinct $\mathrm{T}$ cell subtypes, the predominant types with antitumor activities are $\mathrm{CD}^{+}$cytotoxic T-lymphocytes and $\mathrm{CD} 4^{+} \mathrm{T}$ helper (Th) cells (15). Activated $\mathrm{CT}^{+}$cytotoxic T-lymphocytes mainly serve as immune effectors to induce tumor cell apoptosis, while Th cells, through the production of cytokines, regulate the activation of cytotoxic T-lymphocytes and recruit cells of the innate immune system, including macrophages and mast cells (28). CD4 ${ }^{+}$Th cells consist of two predominant subtypes: Th1 cells characterized by the production of IL-2, IFN- $\gamma$, tumor necrosis factor and Th2 cells characterized by the production of IL-4, IL-5, IL6 and IL-10 (29). Functionally, Th1 cells stimulate cellular immunity, target intracellular pathogens, activate phagocytosis, induce IgG2a, IgG2b and IgG3 antibodies and promote delayed-type hypersensitivity responses (30). By contrast, Th2 cells mainly promote humoral responses, which particularly involve $\operatorname{IgG1}, \operatorname{IgE}$ and $\operatorname{Ig} \mathrm{A}(31)$.

In the paradigm of cancer, myriad studies suggest that Th1-dominant responses are a reflection of anticancer immunity, whereas Th2-dominant responses favor the progression of cancer $(32,33)$. In a mouse model of B cell leukemia/lymphoma, susceptible mice developed a Th2-dominant response, while those developing a Th1-dominant response were more resistant to the disease (34). Similar observations were also noted in mouse models of melanoma, renal cell carcinoma and colon cancer $(35,36)$. Consistent with the mouse models, Th2-predominant phenotypes are also observed in patients with lung cancer, breast cancer, gastric cancer, glioblastoma, renal cell carcinoma, prostate cancer and other types of cancer (32). Collectively, these studies indicate that tilting the balance between the Th1 and Th2 responses towards the former stimulates anticancer immunity, due to the latter promoting

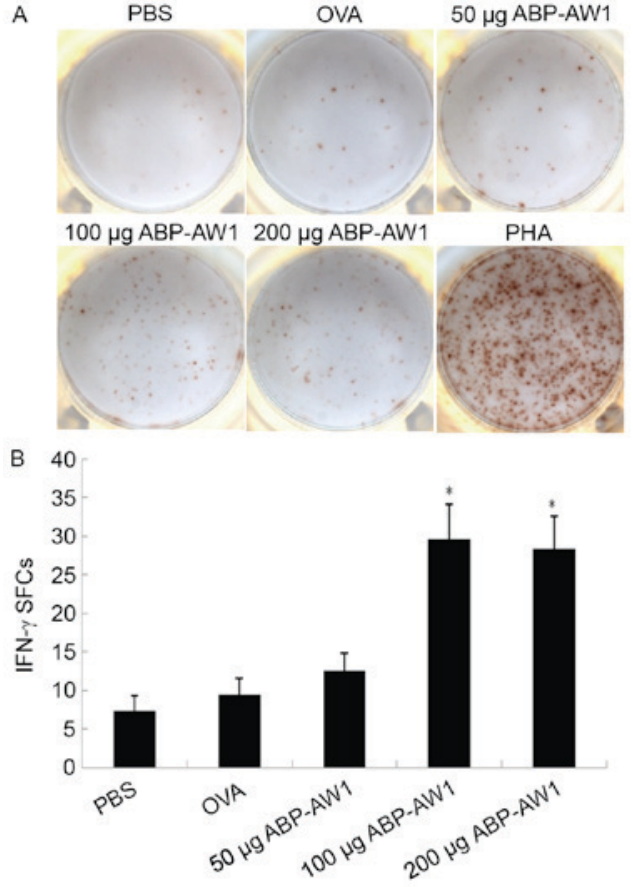

Figure 6. Intermediate and high dose of ABP-AW1 stimulates IFN- $\gamma$ production by splenocytes in response to OVA. Splenocytes isolated from indicated groups of mice were stimulated with OVA. The production of IFN- $\gamma$ was detected by ELISPOT. PHA was used as a positive control. (A) The representative image from each group and (B) quantification of positive signals. ${ }^{*} \mathrm{P}<0.05$, as compared with PBS, OVA or OVA and ABP-AW1 $(50 \mu \mathrm{g})$ group. PHA, phytohemagglutinin; IFN, interferon; OVA, ovalbumin; SFCs, spot-forming cells

the development and progression of cancer; therefore, an ideal immunoadjuvant therapy for cancer vaccine should stimulate the Th1 response, in addition to being non-immunogenic, offering excellent stability, safety, and tolerability, and being cost-effective to produce (14).

Polysaccharides are natural, have low toxicity and are biodegradable (37). They have long been appreciated for their immunomodulating activities and are under intensive investigation for their potential as immunoadjuvant therapies (37). A recent example is the adjuvant therapy $\operatorname{Advax}^{\mathrm{TM}}$, a natural plant-derived polysaccharide that has broad-spectrum boosting effects on B cell, CD4 and CD8 T cell responses, and demonstrated initial success when applied with an influenza (38) or hepatitis B vaccine (39).

In the laboratory, the in vivo immune activity of ABP-AW1 was examined, using OVA as a model antigen. ABP-AW1 was determined to potently induce Th1 responses, as identified by the significantly higher production and secretion of IFN- $\gamma$ from the splenocytes of immunized mice (18). This observation positions ABP-AW1 as an ideal candidate adjuvant therapy for cancer vaccines, and prompted testing of its in vivo efficacy on cancer development. For this purpose, OVA was used as the model antigen and a xenograft tumor model was established via subcutaneous injection of the mouse lymphoma cell line E.G7, which stably expresses OVA. By immunizing the mice with PBS, OVA alone or OVA together with various doses of ABP-AW1, the in vivo efficacy of ABP-AW1 was assessed as an immunoadjuvant therapy. The data demonstrated that OVA by itself was not sufficient to affect tumor growth over time. 
This is consistent with previous findings that the tumor is antigenic, but not immunogenic (40). Similar results were also observed for low-dose (50 $\mu \mathrm{g})$ ABP-AW1 in the present study; however, at intermediate $(100 \mu \mathrm{g})$ and high $(200 \mu \mathrm{g})$ doses, ABP-AW1 was associated with markedly less tumor growth over time and a reduction in the tumor size or weight. This indicated that ABP-AW1 is capable of boosting the anticancer activity of cancer vaccines.

To understand the immune-modulatory mechanisms underlying ABP-AW1, the overall immune activities in mice were assessed. Lymphocytes are key cellular components of the immune system, and their proliferation in response to lymphocyte mitogen or specific antigen is an important measure for host immune activity (41). In the present study, splenocytes were isolated from mice bearing xenograft tumors that had been treated with PBS, OVA alone, or OVA with different doses of ABP-AW1, and the isolated splenocytes were treated in vitro with either ConA (a lymphocyte mitogen) or OVA (a specific tumor antigen). Splenocytes from mice treated with OVA with intermediate- or high-dose ABP-AW1 grew to a much higher number when compared with those from mice treated with PBS, OVA alone, or OVA plus low-dose ABP-AW1. This suggests that co-immunization of the mouse model of lymphoma with OVA and the higher doses of ABP-AW1 not only promotes non-specific lymphocyte proliferation in response to Con A, but also stimulates antigen-specific lymphocyte viability/proliferation.

The second parameter used to measure host immune activity following treatment with OVA and ABP-AW1 was the peripheral $\mathrm{CD}^{+} / \mathrm{CD}^{+} \mathrm{T}$ cell ratio, a marker for immune system status, in which a high $\mathrm{CD} 4^{+} / \mathrm{CD}^{+}$ratio indicates strong immune activity, and a low ratio suggests immune suppression (42). It was determined that OVA combined with intermediate- or high-dose ABP-AW1 was associated with a notably higher peripheral blood $\mathrm{CD} 4^{+} / \mathrm{CD}^{+}$ratio compared with the other groups, also indicating a more active host immune status.

Given that Th1 responses have a crucial role in anticancer immunity, and ABP-AW1 significantly boosts the anticancer activity of a specific tumor antigen, the status of Th1 responses in mice treated with OVA and ABP-AW1 were followed. Specifically, the serum levels of an OVA-specific Th1 antibody (IgG2b) and a Th2 antibody (IgG1) were measured, as well as the production and secretion of Th1 cytokines from splenocytes in response to the specific antigen OVA. In the groups treated with PBS, OVA alone, or OVA and low-dose ABP-AW1, the serum IgG1 levels were higher than the IgG2b levels; that is, the $\operatorname{IgG} 2 \mathrm{~b} / \mathrm{IgG} 1$ ratio was $<1$, indicating that Th2 was predominant in these mice. In groups treated with OVA and intermediate- or high-dose ABP-AW1, the serum IgG2b levels were markedly higher compared with in the other groups, although the serum IgG1 levels were not significantly affected, resulting in $\mathrm{IgG} 2 \mathrm{~b} / \mathrm{IgG} 1$ ratios of $>1$. These data indicate that ABP-AW1 at higher doses boosts Th1 responses, with minimal effect on Th2 responses, and thus shifts the balance from a Th2-predominant phenotype, as observed during cancer development, to a Th1-predominant phenotype, to stimulate anticancer immunity toward the tumor-specific antigen. Consistent with the significant promotion of Th1 responses, significantly higher production of the Th1 cytokine IFN- $\gamma$, and higher levels of IFN- $\gamma$ or IL-2 derived from splenocytes treated with OVA and intermediate- or high-dose ABP-AW1 after in vitro stimulation with OVA were detected, compared with in the other groups.

In conclusion, the evidence demonstrated that ABP-AW1, when incorporated with the tumor-specific antigen OVA into mice bearing OVA-expressing tumors, significantly boosted host immune activity, with specific stimulation of Th1 responses, and notably inhibited tumor growth. It was concluded that ABP-AW1 is a promising adjuvant therapy for cancer immunotherapy.

\section{Acknowledgements}

This study was supported by Qiqihar Municipal Bureau of Science and Technology (grant. no. SFGG-201551) and the Natural Science Foundation of China (grant no. 81173609).

\section{References}

1. Arruebo M, Vilaboa N, Sáez-Gutierrez B, Lambea J, Tres A, Valladares M and González-Fernández A: Assessment of the evolution of cancer treatment therapies. Cancers 3: 3279-3330, 2011.

2. Farkona S, Diamandis EP and Blasutig IM: Cancer immunotherapy: The beginning of the end of cancer? BMC Med 14: 73, 2016.

3. Coley WB: The treatment of malignant tumors by repeated inoculations of erysipelas. With a report of ten original cases. 1893. Clin Orthop Relat Res: 3-11, 1991.

4. Parish CR: Cancer immunotherapy: The past, the present and the future. Immunol Cell Biol 81: 106-113, 2003.

5. Wiemann B and Starnes CO: Coley's toxins, tumor necrosis factor and cancer research: A historical perspective. Pharmacol Ther 64: 529-564, 1994.

6. Drake CG, Lipson EJ and Brahmer JR: Breathing new life into immunotherapy: Review of melanoma, lung and kidney cancer. Nat Rev Clin Oncol 11: 24-37, 2014.

7. Mellman I, Coukos G and Dranoff G: Cancer immunotherapy comes of age. Nature 480: 480-489, 2011.

8. Guo C, Manjili MH, Subjeck JR, Sarkar D, Fisher PB and Wang XY: Therapeutic cancer vaccines: Past, present, and future. Adv Cancer Res 119: 421-475, 2013.

9. Buonaguro L, Petrizzo A, Tornesello ML and Buonaguro FM: Translating tumor antigens into cancer vaccines. Clin Vaccine Immunol 18: 23-34, 2011.

10. Rajput ZI, Hu SH, Xiao CW and Arijo AG: Adjuvant effects of saponins on animal immune responses. J Zhejiang Univ Sci B 8: 153-161, 2007.

11. Jones DE, Palmer JM, Burt AD, Walker C, Robe AJ and Kirby JA: Bacterial motif DNA as an adjuvant for the breakdown of immune self-tolerance to pyruvate dehydrogenase complex. Hepatology 36: 679-686, 2002.

12. Tovey MG and Lallemand C: Adjuvant activity of cytokines. Methods Mol Biol 626: 287-309, 2010.

13. Tefit JN and Serra V: Outlining novel cellular adjuvant products for therapeutic vaccines against cancer. Expert Rev Vaccines 10: 1207-1220, 2011

14. Petrovsky N and Aguilar JC: Vaccine adjuvants: Current state and future trends. Immunol Cell Biol 82: 488-496, 2004.

15. Schietinger A, Philip M, Liu RB, Schreiber K and Schreiber H: Bystander killing of cancer requires the cooperation of CD4(+) and CD8(+) T cells during the effector phase. J Exp Med 207: 2469-2477, 2010

16. Kim HJ and Cantor $\mathrm{H}$ : CD4 T-cell subsets and tumor immunity: The helpful and the not-so-helpful. Cancer Immunol Res 2: 91-98, 2014.

17. Ramberg JE, Nelson ED and Sinnott RA: Immunomodulatory dietary polysaccharides: A systematic review of the literature. Nutr J 9: 54, 2010.

18. Cui L, Sun Y, Xu H, Cong H and Liu J: A polysaccharide isolated from Agaricus blazei Murill (ABP-AW1) as a potential Th1 immunity-stimulating adjuvant. Oncol Lett 6: 1039-1044, 2013. 
19. Liu J and Sun Y: Structural analysis of an alkali-extractable and water-soluble polysaccharide (ABP-AW1) from the fruiting bodies of Agaricus blazei Murill. Carbohydrate Polymers 86: 429-432, 2011

20. Hartmann G, Marschner A, Viveros PR, Stahl-Hennig C, Eisenblatter M, Suh YS, Endres S, Tenner-Racz K, Uberla K, Racz $\mathrm{P}$, et al: $\mathrm{CpG}$ oligonucleotides induce strong humoral but only weak $\mathrm{CD}^{+} \mathrm{T}$ cell responses to protein antigens in rhesus macaques in vivo. Vaccine 23: 3310-3317, 2005.

21. Sjölander A, van't Land B and Lövgren Bengtsson K: Iscoms containing purified Quillaja saponins upregulate both Th1-like and Th2-like immune responses. Cell Immunol 177: 69-76, 1997.

22. Kawase O, Goo YK, Jujo H, Nishikawa Y and Xuan X: Starfish, Asterias amurensis and Asterina pectinifera, as potential sources of Th1 immunity-stimulating adjuvants. J Vet Med Sci 73 227-229, 2011

23. Mountford AP, Fisher A and Wilson RA: The profile of IgG1 and IgG2a antibody responses in mice exposed to Schistosoma mansoni. Parasite Immunol 16: 521-527, 1994.

24. Hanahan D and Weinberg RA: The hallmarks of cancer. Cell 100: 57-70, 2000

25. Hanahan D and Weinberg RA: Hallmarks of cancer: The next generation. Cell 144: 646-674, 2011.

26. Escors D: Tumour immunogenicity, antigen presentation and immunological barriers in cancer immunotherapy. New J Sci 2014: 734515, 2014

27. Dubensky TW Jr and Reed SG: Adjuvants for cancer vaccines. Semin Immunol 22: 155-161, 2010.

28. Knutson KL and Disis ML: Tumor antigen-specific T helper cells in cancer immunity and immunotherapy. Cancer Immuno Immunother 54: 721-728, 2005.

29. Romagnani S: T-cell subsets (Th1 versus Th2). Ann Allergy Asthma Immunol 85: 9-18, 2000.

30. Wang $X$ and Meng D: Innate endogenous adjuvants prime to desirable immune responses via mucosal routes. Protein Cell 6: 170-184, 2015.

31. Sun Y, Liu J, Yu H and Gong C: Isolation and evaluation of immunological adjuvant activities of saponins from the roots of Pulsatilla chinensis with less adverse reactions. Int Immunopharmacol 10: 584-590, 2010.

32. Shurin MR, Lu L, Kalinski P, Stewart-Akers AM and Lotze MT: Th1/Th2 balance in cancer, transplantation and pregnancy. Springer Semin Immunopathol 21: 339-359, 1999.
33. Zamarron BF and Chen W: Dual roles of immune cells and their factors in cancer development and progression. Int J Biol Sci 7: 651-658, 2011.

34. Lee PP, Zeng D, McCaulay AE, Chen YF, Geiler C, Umetsu DT and Chao NJ: T helper 2-dominant antilymphoma immune response is associated with fatal outcome. Blood 90: 1611-1617, 1997.

35. Hu HM, Urba WJ and Fox BA: Gene-modified tumor vaccine with therapeutic potential shifts tumor-specific $\mathrm{T}$ cell response from a type 2 to a type 1 cytokine profile. J Immunol 161: 3033-3041, 1998.

36. Ghosh P, Komschlies KL, Cippitelli M, Longo DL, Subleski J, Ye J, Sica A, Young HA, Wiltrout RH and Ochoa AC: Gradual loss of T-helper 1 populations in spleen of mice during progressive tumor growth. J Natl Cancer Inst 87: 1478-1483, 1995.

37. Tzianabos AO: Polysaccharide immunomodulators as therapeutic agents: Structural aspects and biologic function. Clin Microbiol Rev 13: 523-533, 2000.

38. Honda-Okubo Y, Saade F and Petrovsky N: Advax ${ }^{\mathrm{TM}}$, a polysaccharide adjuvant derived from delta inulin, provides improved influenza vaccine protection through broad-based enhancement of adaptive immune responses. Vaccine 30 : 5373-5381, 2012

39. Saade F, Honda-Okubo Y, Trec S and Petrovsky N: A novel hepatitis $\mathrm{B}$ vaccine containing Advax ${ }^{\mathrm{TM}}$, a polysaccharide adjuvant derived from delta inulin, induces robust humoral and cellular immunity with minimal reactogenicity in preclinical testing. Vaccine 31: 1999-2007, 2013.

40. Blankenstein T, Coulie PG, Gilboa E and Jaffee EM: The determinants of tumour immunogenicity. Nat Rev Cancer 12: 307-313, 2012.

41. Chaplin DD: Overview of the immune response. J Allergy Clin Immunol 125 (Suppl 2): S3-S23, 2010.

42. Serrano-Villar S, Sainz T, Lee SA, Hunt PW, Sinclair E, Shacklett BL, Ferre AL, Hayes TL, Somsouk M, Hsue PY, et al: $\mathrm{HIV}$-infected individuals with low CD4/CD8 ratio despite effective antiretroviral therapy exhibit altered T cell subsets, heightened $\mathrm{CD} 8^{+} \mathrm{T}$ cell activation, and increased risk of non-AIDS morbidity and mortality. PLoS Pathog 10: e1004078, 2014.

(i) () 9 This work is licensed under a Creative Commons Attribution-NonCommercial-NoDerivatives 4.0 International (CC BY-NC-ND 4.0) License. 